\title{
The Burden of Hospital Readmissions among Pediatric Patients with Inflammatory Bowel Disease
}

\author{
Edward L. Barnes, MD, MPH${ }^{1}$, Bharati Kochar, MD¹, Millie D. Long, MD, MPH ${ }^{1,2}$, Christopher F. Martin, MSPH ${ }^{1,2}$, \\ Seth D. Crockett, MD, MPH${ }^{1,2}$, Joshua R. Korzenik, $\mathrm{MD}^{3}$, and Michael D. Kappelman, MD, $\mathrm{MPH}^{2,4}$
}

Objective To evaluate the burden and predictors of hospital readmissions among pediatric patients with inflammatory bowel disease using the Nationwide Readmissions Database.

Study design We performed a retrospective cohort study using 2013 Nationwide Readmissions Database. International Classification of Diseases, Ninth Revision, Clinical Modification codes were used to identify patients $<18$ years with diagnoses of ulcerative colitis (UC) or Crohn's disease (CD). Demographic factors and details of hospitalizations were evaluated using survey procedures in SAS v 9.4 (SAS Institute, Cary, North Carolina). Multivariable logistic regression was used to estimate ORs and $95 \%$ Cls of readmission.

Results Among 2733 hospitalizations (63\% CD, 37\% UC), 611 (22\%) patients were readmitted within 90 days of the index hospitalization. Readmission resulted in weighted estimates of 11440 excess days of hospitalization and total charges of over $\$ 107$ million. For $\mathrm{CD}$, male sex (aOR 1.36, 95\% $\mathrm{Cl} 1.03-1.81$ ) and co-existing anxiety or depression (aOR 1.89, 95\% Cl 1.06-3.40) were associated with increased readmissions, while patients who underwent surgery had decreased readmissions (aOR $0.40,95 \% \mathrm{Cl} 0.24-0.65$ ). In patients with UC, an index admission of $>7$ days was associated with increased readmissions (aOR 1.69, 95\% $\mathrm{Cl} 1.09-2.62$ ).

Conclusions Readmission occurs frequently in children with inflammatory bowel disease and is associated with significant cost and resource burdens. Among patients with $C D$, psychiatric comorbidities such as anxiety and depression are apparent drivers of readmission. (J Pediatr 2017;191:184-9).

ospital readmissions are a key metric associated with quality of healthcare in the US, with the potential to place a great burden on both the patient and the healthcare system. ${ }^{1}$ Recent studies indicate that hospitalizations are increasing for children with inflammatory bowel diseases (IBD) in the US.,3 In addition, a Canadian study demonstrated that pediatric patients with IBD are at greater risk for hospital readmission compared with older patients. ${ }^{4}$ These trends are cause for concern to both patients and payers, as hospitalization of pediatric patients with IBD has been associated with a significant cost burden ${ }^{5-7}$ and hospitalization has been associated with decreased health-related quality of life in other populations of children with chronic diseases. ${ }^{8}$

Despite these concerns, there remains a paucity of literature examining the factors associated with hospital readmissions in children with Crohn's disease (CD) and ulcerative colitis (UC). Studies of postsurgical readmissions in children with IBD in-

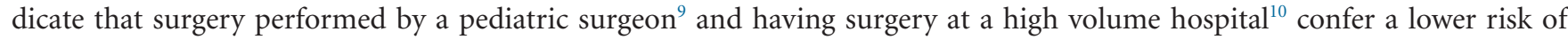
postoperative readmission. However, the exact role that healthcare disparities, racial differences in phenotypes, or other confounding factors play in these risks for readmission is uncertain. ${ }^{11}$ Single center experiences from American adults with IBD suggest that factors such as severe disease, poorly controlled pain and depression, and admission to a teaching service are associated with a higher likelihood of readmission, ${ }^{9-11}$ but it is unknown if these same factors are important in children with IBD.

Our objectives were to use the Nationwide Readmissions Database (NRD) to describe the overall burden of readmission in patients with IBD, including the total charges associated with readmission, and analyze the specific factors associated with hospital readmission within 90 days.

\section{Methods}

We conducted a retrospective cohort study using 2013 data from the NRD, a resource of the Healthcare Cost and Utilization Project (HCUP) State Inpatient

$\begin{array}{ll}\text { CD } & \text { Crohn's disease } \\ \text { HCUP } & \text { Healthcare Cost and Utilization Project } \\ \text { IBD } & \text { Inflammatory bowel disease } \\ \text { ICD-9-CM } & \text { International Classification of Diseases, Ninth Revision, Clinical Modification } \\ \text { LOS } & \text { Length of stay } \\ \text { NRD } & \text { Nationwide Readmissions Database } \\ \text { UC } & \text { Ulcerative colitis }\end{array}$

From the ${ }^{1}$ Division of Gastroenterology and Hepatology, University of North Carolina at Chapel Hill; ${ }^{2}$ Center for Gastrointestinal Biology and Disease, Chapel Hill, NC ${ }^{3}$ Division of Gastroenterology, Hepatology, and Endoscopy, Brigham and Women's Hospital, Boston, MA; and ${ }^{4}$ Division of Pediatric Gastroenterology and Hepatology, University of North Carolina at Chapel Hill, Chapel Hill, NC

Supported by the National Institutes of Health (T32DK07634 [to B.K.] and KL2-RR025746 [to S.C.]). The authors declare no conflicts of interest. 
Databases. Designed to be nationally representative in the adult population, the NRD is a publicly available all-payer inpatient database from the US that contains discharge data from 22 geographically dispersed states accounting for $49.3 \%$ of all US hospitalizations. Weights were assigned to each admission by HCUP to allow for national readmission analyses. The NRD contains over 100 clinical and nonclinical variables, ${ }^{12}$ including outcomes such as hospital length of stay (LOS), readmission, and total charges. The NRD also contains up to 15 diagnostic and procedure codes per admission based on International Classification of Diseases, Ninth Revision, Clinical Modification (ICD-9-CM) coding. This study was determined exempt from review by the University of North Carolina Institutional Review Board, given the deidentified nature of the data.

We examined discharge data from patients age 17 years and younger. Patients were determined to have IBD if they had a primary or secondary ICD-9-CM diagnosis code indicating CD (555.xx) or UC (556.xx) on the index admission. Given that patients with UC undergoing colectomy could have a scheduled readmission for repeat operation such as ileal pouchanal anastomosis within the 90 day follow-up for readmission, we excluded any patient with an ICD-9-CM code for colectomy. Approximately $1 \%$ of patients were given discharge codes for both CD and UC $(\mathrm{n}=28)$, and to minimize misclassification of subgroups of IBD, we excluded those patients.

The primary outcome of interest, hospital readmission within 90 days of the index hospitalization, was determined using a unique identifier specific to the NRD to link discharge data of individual patients. We also evaluated the primary ICD-9CM diagnosis code on readmission, LOS during all hospitalizations, and the total charges associated with readmission.

Patient demographics included age, sex, and primary insurance/payer for the index hospitalization. We used ICD9-CM diagnosis codes to identify additional comorbidities or factors suggested to be independent predictors or confounders of the relationship between IBD and hospital readmission. Given prior suggestions in adult populations that modifiable risk factors such as depression (ICD-9-CM 296.2, $296.3,298.0,300.4,311)$, chronic pain $(338.2,338.4)$, and anxiety $(300,300.2)$ play a significant role in risk for readmission we made the a priori decision to include these covariates in our analyses. We used ICD-9-CM definitions for anxiety and depression that have previously been validated in IBD using administrative data. ${ }^{13}$ In addition, the diagnosis coding used to identify admissions with Clostridium difficile infection (ICD-9-CM 008.45) has previously been used in HCUP databases. ${ }^{14}$ IBD-related surgery was included in the models for CD only. For patients with CD, ICD-9-CM coding was used to identify potential complications of CD including small bowel obstruction and fistulizing disease (ICD-9-CM codes listed in Table I; available at www.jpeds.com).

In the NRD, hospital teaching status is defined as "metropolitan teaching," "metropolitan nonteaching," and "rural." HCUP defines an institution as a teaching hospital if it has an American Medical Association-approved residency program, if the institution is a member of the Council of Teaching
Hospitals, or based on the ratio of full-time equivalent interns and residents to hospital beds ( 0.25 or higher). ${ }^{12}$ In other HCUP databases such as the Nationwide Inpatient Sample and the Kids' Inpatient Database, approximately $98 \%$ of patients with IBD admitted to rural hospitals are admitted to nonteaching hospitals, ${ }^{15}$ and HCUP indicates that rural teaching hospitals are rare. ${ }^{12}$ For our analyses, we collapsed rural and metropolitan nonteaching into 1 category and refer to metropolitan teaching hospitals as "teaching." We also analyzed hospitals based on annual volume of patients with IBD, using thresholds previously established in the Kids' Inpatient Database, ${ }^{7}$ where those hospitals with an annual volume of IBD discharges of $>20$ were defined as high volume IBD centers.

\section{Statistical Analyses}

All analyses were performed using survey procedures in SAS v 9.4 (SAS Institute, Cary, North Carolina). Data were weighted to reflect estimates of the national population, including the means and proportions presented. The $\chi^{2}$ test and Student $t$ test were used to compare proportions and continuous variables, respectively. We used logistic regression modeling to estimate the unadjusted odds of a readmission within 90 days of the index hospitalization for the covariates of interest. In all logistic regression models, we made the a priori decision to include only patients with Medicaid (33\% of all admissions) or private insurance ( $60 \%$ of all admissions) as their primary payer for the admission, given concerns that there may be significant overlap between the "other" insurance provider group and the Medicaid group. In prespecified sensitivity analyses, we included all patients, regardless of payer status, to assess for any differences in outcomes when patients with other payers were included.

Based on clinical knowledge and previously reported risk factors for readmission among adult populations of patients with IBD, we then evaluated the association between risk factors and readmission within 90 days using multivariable logistic regression. All factors included in the models were chosen due to their clinical relevance and were identified a priori, including payer and hospital specific factors. For all analyses, diagnoses of anxiety or depression were collapsed into one variable. Any covariate with $<11$ patients was excluded from the multivariable models. ORs and 95\% CIs are presented. A 2-tailed $P$ value of .05 was chosen as the threshold for statistical significance for all tests.

\section{Results}

A total of 2733 (63\% CD, 37\% UC) patients with IBD were identified in the NRD, of which 611 (22\%) required readmission within 90 days of the index hospitalization. Using the NRD weighting, these represented 8632 nationwide admissions and 1845 nationwide readmissions among pediatric patients with IBD in 2013. Hospital readmission resulted in an additional 3788 days of hospitalization for those affected, representing a weighted estimate of 11440 excess days in the national population of patients with IBD. Among patients with CD, the median LOS for the first readmission was 3.69 days 
Table II. Baseline clinical and demographic characteristics of pediatric patients with IBD in the 2013 NRD

\begin{tabular}{|c|c|c|c|c|}
\hline & \multicolumn{2}{|c|}{$\begin{array}{c}\text { CD } \\
n=1733\end{array}$} & \multicolumn{2}{|c|}{$\begin{array}{c}\text { UC } \\
n=1000\end{array}$} \\
\hline & $\mathbf{n}$ & $\%$ & $\mathbf{n}$ & $\%$ \\
\hline \multicolumn{5}{|l|}{ Age $(y)$} \\
\hline$\leq 12$ & 451 & 26 & 416 & 42 \\
\hline 13-17 & 1282 & 74 & 684 & 68 \\
\hline Male sex & 979 & 56 & 497 & 50 \\
\hline Anxiety or depression & 101 & 6 & 43 & 4 \\
\hline Chronic pain & $<11$ & 1 & $<11$ & 1 \\
\hline $\begin{array}{l}\text { Teaching hospital on index } \\
\text { admission }\end{array}$ & 1549 & 89 & 869 & 87 \\
\hline \multicolumn{5}{|l|}{$\begin{array}{l}\text { Hospital volume on index } \\
\text { admission }\end{array}$} \\
\hline$\leq 20$ IBD admissions per y & 459 & 28 & 302 & 31 \\
\hline$>20$ IBD admissions per y & 1274 & 72 & 698 & 69 \\
\hline \multicolumn{5}{|l|}{ Payer/insurance } \\
\hline Medicaid & 549 & 32 & 375 & 38 \\
\hline Private insurance & 1065 & 61 & 575 & 58 \\
\hline Other & 119 & 7 & 78 & 8 \\
\hline C difficile infection & 87 & 5 & 67 & 7 \\
\hline Need for IBD-related surgery & 225 & 13 & $\mathrm{~N} / \mathrm{A}$ & $\mathrm{N} / \mathrm{A}$ \\
\hline \multicolumn{5}{|l|}{ LOS for index admission (d) } \\
\hline$\leq 3$ & 796 & 46 & 390 & 39 \\
\hline $4-7$ & 597 & 34 & 353 & 35 \\
\hline$>7$ & 340 & 20 & 257 & 26 \\
\hline Presence of a fistula & 60 & 3 & $\mathrm{~N} / \mathrm{A}$ & N/A \\
\hline Small bowel obstruction & 226 & 13 & $\mathrm{~N} / \mathrm{A}$ & N/A \\
\hline
\end{tabular}

(IQR1.95-6.58) compared with a median LOS of 4.53 days (IQR 2.41-8.84) for patients with UC $(P=.057)$. Among patients with $\mathrm{CD}$ requiring readmission, $85 \%$ had a diagnosis of $\mathrm{CD}$ as a primary diagnosis. The other most common primary or secondary diagnoses on readmission among patients with $\mathrm{CD}$ were anemia (3.3\%), abnormal loss of weight (2\%), dehydration $(1.5 \%)$, and $C$ difficile infection (0.6\%). Among patients with UC requiring readmission, $89 \%$ had UC as a primary diagnosis. The other most common primary or secondary diagnoses on readmission among patients with UC were anemia (13\%), abnormal loss of weight (5.1\%), C difficile infection (2.5\%), and dehydration (2.5\%). All ICD-9-CM codes for comorbid diagnoses are listed in Table I.

The baseline demographics of the CD and UC populations are depicted in Table II. There was no significant difference in readmission if the diagnosis of $\mathrm{CD}$ was listed as the primary or secondary diagnosis (22\% vs $17 \%, P=.105)$. Although the raw percentage of patients with a primary diagnosis of UC requiring readmission was also greater than that of patients with a secondary diagnosis of UC, there was no statistically significant difference in readmission within 90 days ( $25 \%$ vs $17 \%, P=.066)$.

Among patients with IBD who were readmitted within 90 days, the median total charges for the first readmission were \$31 282 (IQR \$16 491-\$62 496). There was no significant difference when comparing the median total charges for the first readmission in patients with UC to patients with CD ( $\$ 33712$ vs $\$ 30387, P=.270$ ). When aggregated among all pediatric patients with IBD in the NRD, the total charges attributed to the first readmission within 90 days were \$35 698897 with a weighted estimate of \$107 820643 in the national population of pediatric patients with IBD. Among readmissions of patients with $\mathrm{CD}, 78 \%$ were identified as nonelective readmissions. Sixty-seven percent of patients with UC requiring readmission were reported to have a nonelective readmission.

\section{Factors Associated with Readmission in CD}

In univariate analysis of patients with $\mathrm{CD}$, those patients requiring readmission were more likely to be male, have a preexisting psychiatric diagnosis of anxiety or depression (Figure; available at www.jpeds.com), and were less likely to have undergone an IBD related surgery during the index admission (Table III). There was no significant difference in readmission

Table III. Univariate comparison of characteristics of pediatric patients with CD in the 2013 NRD, including unadjusted odds of readmission

\begin{tabular}{|c|c|c|c|c|c|c|}
\hline & \multicolumn{2}{|c|}{$\begin{array}{c}\text { No readmission } \\
n=1364\end{array}$} & \multicolumn{2}{|c|}{$\begin{array}{l}\text { Readmission } \\
n=369\end{array}$} & \multirow[t]{2}{*}{$\begin{array}{l}\text { Unadjusted OR } \\
(95 \% \mathrm{CI})\end{array}$} & \multirow[t]{2}{*}{$P$ value } \\
\hline & $\mathbf{n}$ & $\%$ & $\mathbf{n}$ & $\%$ & & \\
\hline Age (y) & & & & & & .183 \\
\hline$\leq 12$ & 371 & 27 & 80 & 22 & Reference & \\
\hline $13-17$ & 993 & 73 & 289 & 78 & $1.22(0.88-1.70)$ & \\
\hline Male sex & 756 & 55 & 223 & 60 & $1.33(1.01-1.75)$ & .004 \\
\hline Anxiety or depression & 73 & 5 & 28 & 8 & $1.99(1.15-3.45)$ & .028 \\
\hline Index admission to teaching hospital & 1208 & 88 & 341 & 92 & $1.35(0.85-2.14)$ & .206 \\
\hline Index admission to high volume IBD center & 980 & 72 & 294 & 74 & $1.12(0.80-1.55)$ & .509 \\
\hline Payer/insurance & & & & & & .087 \\
\hline Medicaid & 414 & 30 & 135 & 37 & $1.28(0.97-1.69)$ & \\
\hline Private insurance & 857 & 63 & 208 & 56 & Reference & \\
\hline C difficile infection & 61 & 5 & 26 & 7 & $1.50(0.87-2.57)$ & .200 \\
\hline Need for IBD-related surgery & 196 & 14 & 29 & 8 & $0.49(0.31-0.78)$ & .003 \\
\hline LOS for index hospitalization (d) & & & & & & .057 \\
\hline$\leq 3$ & 640 & 47 & 156 & 42 & $0.83(0.60-1.13)$ & \\
\hline $4-7$ & 471 & 35 & 126 & 34 & Reference & \\
\hline$>7$ & 253 & 19 & 87 & 24 & $1.22(0.84-1.76)$ & \\
\hline Presence of a fistula & 48 & 4 & 12 & 3 & $0.84(0.38-1.88)$ & .754 \\
\hline Small bowel obstruction & 173 & 13 & 53 & 14 & $1.33(0.88-2.02)$ & .067 \\
\hline
\end{tabular}


Table IV. Multivariable analysis, odds of readmission among pediatric patients with CD or UC in the 2013 $\mathrm{NRD}^{*}$

\begin{tabular}{lcc}
\hline & $\begin{array}{c}\text { CD } \\
\text { aOR } \mathbf{9 5 \%} \mathbf{~ C l})\end{array}$ & $\begin{array}{c}\text { UC } \\
\text { aOR } \mathbf{( 9 5 \% ~ C l )}\end{array}$ \\
\hline Age (y) & Reference & Reference \\
$\leq 12$ & $1.32(0.94-1.85)$ & $0.89(0.62-1.28)$ \\
$13-17$ & $1.36(1.03-1.81)$ & $0.81(0.58-1.14)$ \\
Male Sex & $1.89(1.06-3.40)$ & $1.57(0.69-3.54)$ \\
Anxiety or depression & $1.36(0.80-2.32)$ & $1.32(0.74-2.37)$ \\
Index admission to teaching hospital & $1.02(0.70-1.49)$ & $1.27(0.84-1.92)$ \\
Index admission to high volume & & \\
IBD center & & \\
Payer/insurance & $1.23(0.92-1.63)$ & $1.19(0.84-1.69)$ \\
Medicaid & Reference & Reference \\
Private insurance & $0.40(0.24-0.65)$ & N/A \\
Need for IBD-related surgery & & \\
LOS for index admission (d) & $0.79(0.57-1.08)$ & $1.05(0.70-1.57)$ \\
$\leq 3$ & Reference & Reference \\
$4-7$ & $1.28(0.87-1.89)$ & $1.69(1.09-2.62)$ \\
$>7$ & &
\end{tabular}

if the diagnosis of $\mathrm{CD}$ was listed as the primary or secondary diagnosis $(22 \%$ vs $17 \%, P=.105)$. In multivariable analysis, male sex (aOR 1.36, 95\% CI 1.03-1.81) and co-existing anxiety or depression (aOR 1.89, 95\% CI 1.06-3.40) were associated with a significant increase in odds of readmission within 90 days of index hospitalization (Table IV). In addition, the need for IBD related surgery was associated with decreased odds of readmission within 90 days (aOR $0.40,95 \%$ CI 0.24-0.65). In the prespecified sensitivity analysis including all payer sources, there was no change in the final results.

\section{Factors Associated with Readmission in UC}

In univariate analysis, patients with UC requiring readmission were more likely to be admitted to teaching hospitals, more likely to be admitted to high volume IBD centers, and demonstrated longer LOS on the index admission when compared with patients who did not require readmission (Table V). When patients with UC were evaluated in multivariable analysis, only a LOS of $>7$ days during the index admission was associated with an increased odds of readmission within 90 days (aOR 1.69, 95\% CI 1.09-2.62, Table IV). Although not significant, an increased odds of readmission within 90 days was suggested with a comorbid condition of anxiety or depression (aOR 1.57, 95\% CI 0.69-3.54), index admission to a teaching hospital (aOR 1.32, 95\% CI 0.74-2.37), and index admission to a high volume IBD center (aOR 1.27, 95\% CI 0.84-1.69). A sensitivity analysis was also performed including all payer sources, which demonstrated no change in the final results.

\section{Discussion}

Based on this US nationwide study of pediatric patients with $\mathrm{UC}$ and $\mathrm{CD}$, the burden of readmissions is substantial. Over $20 \%$ of patients with IBD required a repeat hospitalization within 90 days of the index admission. These readmissions resulted in a national weighted estimate of over 11000 excess hospital days and approximately $\$ 107$ million total charges in the pediatric IBD population. Among patients with $\mathrm{CD}$, male sex and a coexisting diagnosis of anxiety or depression were associated with significantly increased odds of readmission within 90 days. In patients with UC, an increased LOS during the index admission was associated with increased odds of readmission.

The majority of the literature evaluating risk factors for readmission among patients with IBD has evaluated the adult population. Prior single center studies evaluating adults with IBD have associated an increased risk of hospital readmission with factors such as chronic pain and depression. ${ }^{16}$ Both anxiety and depression are increased among patients with IBD compared with other disease states and the general population. ${ }^{17-19}$ In addition, anxiety has been associated with worse outcomes among pediatric patients with $\mathrm{IBD}^{20}$ and

Table V. Univariate comparison of characteristics of pediatric patients with UC in the $2013 \mathrm{NRD}$, including unadjusted odds of readmission

\begin{tabular}{|c|c|c|c|c|c|c|}
\hline & \multicolumn{2}{|c|}{$\begin{array}{l}\text { No readmission } \\
n=758\end{array}$} & \multicolumn{2}{|c|}{$\begin{array}{l}\text { Readmission } \\
n=242\end{array}$} & \multirow{2}{*}{$\begin{array}{l}\text { Unadjusted OR } \\
(95 \% \mathrm{Cl})\end{array}$} & \multirow[b]{2}{*}{$P$ value } \\
\hline & $\mathbf{n}$ & $\%$ & $\mathbf{n}$ & $\%$ & & \\
\hline Age (y) & & & & & & .652 \\
\hline$\leq 12$ & 239 & 32 & 77 & 32 & Reference & \\
\hline $13-17$ & 519 & 68 & 165 & 68 & $0.86(0.60-1.24)$ & \\
\hline Male sex & 381 & 50 & 116 & 48 & $0.78(0.56-1.10)$ & .109 \\
\hline Anxiety or depression & 29 & 4 & 14 & 6 & $1.66(0.77-3.54)$ & .123 \\
\hline Index admission to teaching hospital & 645 & 85 & 224 & 93 & $1.73(1.01-2.95)$ & .005 \\
\hline Index admission to high volume IBD hospital & 502 & 66 & 196 & 76 & $1.46(1.01-2.12)$ & .045 \\
\hline Payer/insurance & & & & & & .036 \\
\hline Medicaid & 256 & 34 & 91 & 38 & $1.28(0.91-1.82)$ & \\
\hline Private insurance & 444 & 59 & 131 & 54 & Reference & \\
\hline C difficile infection & 56 & 7 & 11 & 5 & $0.52(0.25-1.10)$ & .088 \\
\hline LOS for index admission (d) & & & & & & .002 \\
\hline$\leq 3$ & 306 & 40 & 84 & 35 & $1.02(0.67-1.53)$ & \\
\hline $4-7$ & 275 & 36 & 78 & 32 & Reference & \\
\hline$>7$ & 177 & 23 & 80 & 33 & $1.82(1.18-2.80)$ & \\
\hline
\end{tabular}


increased resource utilization among adult patients with IBD. ${ }^{21,22}$ Although the impact of these psychological comorbidities has been evaluated in multiple studies, a majority of patients with $\mathrm{UC}$ or CD may not receive adequate psychosocial support in the scheme of their overall treatment for IBD. ${ }^{23}$ Among patients with $\mathrm{CD}$ in our population, a co-existing diagnosis of anxiety or depression was associated with readmission within 90 days in both the unadjusted analysis and adjusted analyses. Although the association between a co-existing diagnosis of anxiety or depression and readmission was not significant in patients with UC, the magnitude of the effect estimate would seem to suggest that this relationship may also exist among patients with UC. Although this study may have been underpowered to identify a significant relationship, further study of this potential association is indicated in patients with UC, as are efforts to better identify and treat psychological comorbidities among inpatients with IBD.

Among patients with IBD, chronic pain has also been associated with significant effects on quality of life. ${ }^{24}$ Less than 11 patients in both populations had a co-existing diagnosis of chronic pain, and, thus, chronic pain was not used in our analyses of patients with UC or CD. In a study of adult inpatients with IBD, a diagnosis of CD was associated with increased use of narcotics despite a lack of association with disease activity on objective evaluation such as radiographic imaging or endoscopy. ${ }^{25}$ Taken together, continued examination of the role of pain among pediatric patients with IBD seems essential, as this population was underpowered to evaluate the relationship that chronic pain may play in readmission among pediatric patients with IBD.

In both univariate and multivariable analysis of pediatric patients with CD, the need for IBD-related surgery was a strong negative predictor for readmission within 90 days among pediatric patients. This may indicate that surgery offers either a temporary or definitive successful therapeutic option in patients with $\mathrm{CD}$, as only $8 \%$ of patients requiring an IBD related surgery during the index admission were readmitted within 90 days. In a prior evaluation of children with CD and intraabdominal abscesses, Dotson et $\mathrm{al}^{26}$ found that $64 \%$ of patients with intra-abdominal abscesses treated with medical management or percutaneous drainage ultimately required surgical intervention within one year. Although preservation of intestinal length remains a critical focus in pediatric patients with $\mathrm{CD}$, definitive management with resection in cases of perforation and abscess may be key in allowing a patient to resume school and age appropriate activities more quickly ${ }^{27}$ while potentially decreasing the risk for readmission.

Among patients with UC, a longer LOS during the index admission was associated with an increased risk of readmission within 90 days. Although not significant, we also observed that hospitalization at a teaching hospital seemed to be associated with increased odds of readmission. We speculate that both of these may represent indirect markers of disease severity. However, the NRD does not contain sufficient clinical data (laboratory data, medication use, or phenotypic information) to fully explore the relationships between disease severity, hospital characteristics, and readmission rates.
The NRD is designed to be a nationally representative sample of hospitalizations within the US. However, as the majority of hospitalizations are for adult patients, it is unknown whether the NRD is truly representative of the entire population of children with IBD in the US. Nevertheless, the NRD remains the largest, most geographically diverse source of hospitalization and readmission data available in the US. There are other limitations in our study as well. As noted, the NRD does not contain laboratory data or clinical data regarding the severity of an individual patient's disease at the time of hospitalization, and, thus, aside from surrogate indicators such as LOS, our ability to evaluate the relationship between the severity of disease and odds of readmission is limited. Although we evaluated variables such as the presence of a fistula or a small bowel obstruction, the potential remains that these diagnoses may not be coded as accurately as a primary diagnosis of CD.

In addition, the NRD does not contain information on race. This is an important limitation given prior literature demonstrating a shorter time to readmission and a higher probability of readmission among black children with IBD when compared with white children. ${ }^{11}$ There is also the potential for misclassification of those factors associated with readmission when using large databases such as the NRD. Because of the high likelihood that active IBD is the primary driver of many index admissions, the potential exists that comorbid conditions such as anxiety, depression, and chronic pain may be less likely to be coded. This could lead to an underestimation of the influence that these comorbid diseases have on readmission, even when using validated algorithms for casefinding. We were also unable to evaluate provider factors that may have contributed to readmission, including the impact that factors such as early discharge from the index hospitalization play in readmission. Although future interest in decreasing readmission will likely focus on multidisciplinary approaches to discharge planning, a continued focus on provider decision making during the index hospitalization will remain critical in efforts to improve outcomes among hospitalized children with IBD.

In conclusion, using a nationwide database, we identified a significant economic burden associated with hospital readmission among pediatric patients with UC and CD. Among patients with $\mathrm{CD}$, psychiatric comorbidities of anxiety or depression and male sex were associated with increased odds of readmission within 90 days. Those patients with UC who experienced longer LOS during their index admission were at increased odds of readmission within 90 days. These findings demonstrate the significant economic burden of readmission in this population and given the paucity of literature evaluating the burden of hospital readmission among children with IBD, may lead to future studies to better identify risk factors associated with readmission.

Submitted for publication May 24, 2017; last revision received Jul 6, 2017; accepted Aug 16, 2017

Reprint requests: Edward L. Barnes, MD, MPH, Division of Gastroenterology and Hepatology, University of North Carolina at Chapel Hill, 130 Mason Farm Rd, Bioinformatics Building, CB \#7080, Chapel Hill, NC 27599-7080. E-mail: edward_barnes@med.unc.edu 


\section{References}

1. Jencks SF, Williams MV, Coleman EA. Rehospitalizations among patients in the Medicare fee-for-service program. N Engl J Med 2009;360:1418-28.

2. Sandberg KC, Davis MM, Gebremariam A, Adler J. Increasing hospitalizations in inflammatory bowel disease among children in the United States, 1988-2011. Inflamm Bowel Dis 2014;20:1754-60.

3. deBruyn JCC, Soon IS, Hubbard J, Wrobel I, Panaccione R, Kaplan GG. Nationwide temporal trends in incidence of hospitalization and surgical intestinal resection in pediatric inflammatory bowel diseases in the united states from 1997 to 2009. Inflamm Bowel Dis 2013;19:242332.

4. Nguyen GC, Bollegala N, Chong CA. Factors associated with readmissions and outcomes of patients hospitalized for inflammatory bowel disease. Clin Gastroenterol Hepatol 2014;12:1897-904, e1.

5. Heaton PC, Tundia NL, Schmidt N, Wigle PR, Kelton CML. National burden of pediatric hospitalizations for inflammatory bowel disease. J Pediatr Gastroenterol Nutr 2012;54:477-85.

6. Pant C, Anderson MP, Deshpande A, Altaf MA, Grunow JE, Atreja A, et al. Health care burden of Clostridium difficile infection in hospitalized children with inflammatory bowel disease. Inflamm Bowel Dis 2013;19:10805.

7. Pant C, Deshpande A, Sferra TJ, Almadhoun O, Batista D, Pervez A, et al. Pediatric hospitalizations for inflammatory bowel disease based on annual case volume: results from the Kids' Inpatient Database 2012. J Investig Med 2017;65:94-6.

8. Naughton MJ, Ruggiero AM, Lawrence JM, Imperatore G, Klingensmith GJ, Waitzfelder B, et al. Health-related quality of life of children and adolescents with type 1 or type 2 diabetes mellitus: SEARCH for Diabetes in Youth Study. Arch Pediatr Adolesc Med 2008;162:649-57.

9. Jan AS, Slap G. Variation in surgical outcomes for adolescents and young adults with inflammatory bowel disease. Pediatrics 2013;131(Suppl 1):S819.

10. Kelley-Quon LI, Tseng C, Jen HC, Ziring DA, Shew SB. Postoperative complications and health care use in children undergoing surgery for ulcerative colitis. J Pediatr Surg 2012;47:2063-70.

11. Dotson JL, Kappelman MD, Chisolm DJ, Crandall WV. Racial disparities in readmission, complications, and procedures in children with Crohn's disease. Inflamm Bowel Dis 2015;21:801-8.

12. Introduction to the HCUP Nationwide Readmission Database (NRD) 2016 [Internet]. https://www.hcup-us.ahrq.gov/db/nation/nrd/NRD _Introduction_2013.jsp\#intro. Accessed January 1, 2016.

13. Marrie RA, Walker JR, Graff LA, Lix LM, Bolton JM, Nugent Z, et al. Performance of administrative case definitions for depression and anxiety in inflammatory bowel disease. J Psychosom Res 2016;89:107-13.
14. Kassam Z, Cribb Fabersunne C, Smith MB, Alm EJ, Kaplan GG, Nguyen GC, et al. Clostridium difficile associated risk of death score (CARDS): a novel severity score to predict mortality among hospitalised patients with C. difficile infection. Aliment Pharmacol Ther 2016;43:725-33.

15. Barnes EL. Unpublished data, kids' inpatient database nationwide inpatient sample. 2017.

16. Allegretti JR, Borges L, Lucci M, Chang M, Cao B, Collins E, et al. Risk factors for rehospitalization within 90 days in patients with inflammatory bowel disease. Inflamm Bowel Dis 2015;21:2583-9.

17. Kappelman MD, Long MD, Martin C, Dewalt DA, Kinneer PM, Chen W, et al. Evaluation of the patient-reported outcomes measurement information system in a large cohort of patients with inflammatory bowel diseases. Clin Gastroenterol Hepatol 2014;12:1315-23, e2.

18. Loftus E V, Guérin A, Yu AP, Wu EQ, Yang M, Chao J, et al. Increased risks of developing anxiety and depression in young patients with Crohn's disease. Am J Gastroenterol 2011;106:1670-7.

19. Mikocka-Walus A, Knowles SR, Keefer L, Graff L. Controversies revisited. Inflamm Bowel Dis 2016;22:752-62.

20. Reigada LC, Hoogendoorn CJ, Walsh LC, Lai J, Szigethy E, Cohen BH, et al. Anxiety symptoms and disease severity in children and adolescents with Crohn disease. J Pediatr Gastroenterol Nutr 2015;60:30-5.

21. Ramos-Rivers C, Regueiro M, Vargas EJ, Szigethy E, Schoen RE, Dunn $\mathrm{M}$, et al. Association between telephone activity and features of patients with inflammatory bowel disease. Clin Gastroenterol Hepatol 2014;12:98694, el.

22. Click B, Ramos Rivers C, Koutroubakis IE, Babichenko D, Anderson AM, Hashash JG, et al. Demographic and clinical predictors of high healthcare use in patients with inflammatory bowel disease. Inflamm Bowel Dis 2016;22:1442-9.

23. Bennebroek Evertsz' F, Thijssens NAM, Stokkers PCF, Grootenhuis MA, Bockting CLH, Nieuwkerk PT, et al. Do inflammatory bowel disease patients with anxiety and depressive symptoms receive the care they need? J Crohns Colitis 2012;6:68-76.

24. Morrison G, Van Langenberg DR, Gibson SJ, Gibson PR. Chronic pain in inflammatory bowel disease. Inflamm Bowel Dis 2013;19:1210-7.

25. Long MD, Barnes EL, Herfarth HH, Drossman D. Narcotic use for inflammatory bowel disease and risk factors during hospitalization. Inflamm Bowel Dis 2012;18:869-76.

26. Dotson JL, Nwomeh B, Andridge R, Anderson SE, Crandall WV. Variation in management of intra-abdominal abscesses in children with Croh's disease. Inflamm Bowel Dis 2013;19:818-25.

27. Pfefferkorn MD, Marshalleck FE, Saeed SA, Splawski JB, Linden BC, Weston BF. NASPGHAN clinical report on the evaluation and treatment of pediatric patients with internal penetrating Crohn disease: intraabdominal abscess with and without fistula. J Pediatr Gastroenterol Nutr 2013;57:394400. 


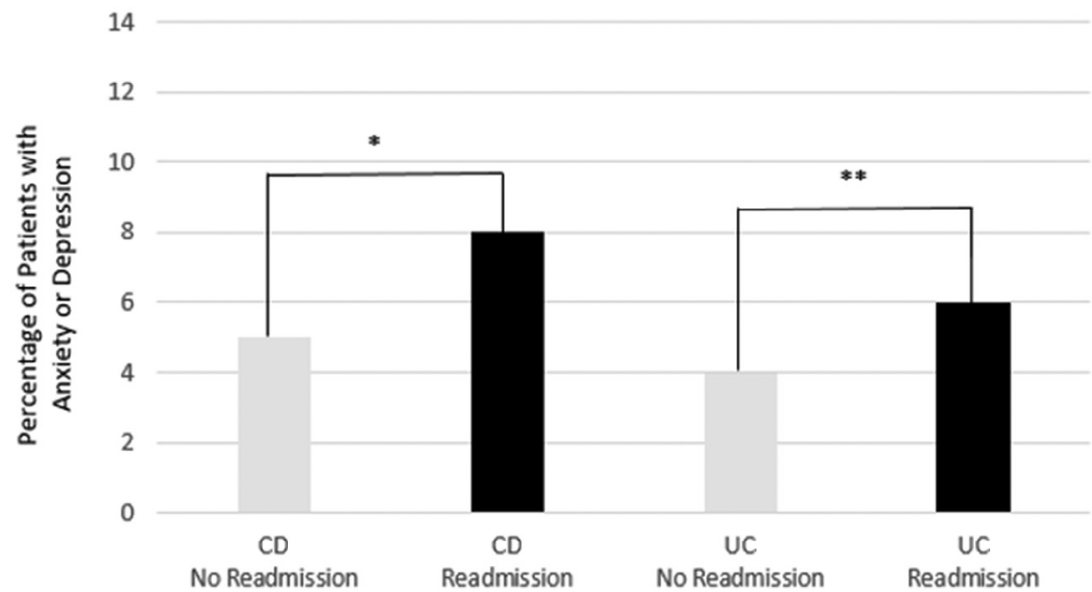

Figure. Comparison of the percentage of patients with a coexisting diagnosis of anxiety or depression stratified by readmission status and primary diagnosis of $\mathrm{CD}$ or UC, ${ }^{\star} P=.028,{ }^{\star \star} P=.123$.

Table I. ICD-9-CM diagnosis codes used in the analysis of 90-day readmissions among patients with $\mathrm{CD}$ and $\mathrm{UC}$

\section{Diagnoses}

ICD-9-CM Code

C difficile infection

Small intestinal resection

Colectomy

Other abdominal operations

Dehydration

Anemia

Depression

Anxiety

Chronic pain

Fistula

CD

UC

Intestinal obstruction

Abnormal weight loss

008.45

$45.6 \times$

$45.7 x, 45.8 x$

54.xx

276.51

$280.0,280.9,281.9,285.1,285.29,285.9$

296.2, 296.3, 298,0 300.4, 311

$300,300.2$

338.2 or 338.4

$537.4,569.81,619.1$

$555 . x x$

$556 . x x$

$560.81,560.89,560.9$

783.21 